\title{
RESPIRATORY MUSCLE ACTIVITY AFTER DEEP NEUROMUSCULAR BLOCKADE AND REVERSEL WITH SUGAMMADEX
}

\author{
Derek Garne ${ }^{1}$, Chung Yin Cheng ${ }^{1}$, Koen Janssens ${ }^{2}$, Sabine Maes ${ }^{1}$, Vera Saldien ${ }^{1}$, Tom Schepens ${ }^{13}$
}

Department of ${ }^{1}$ Anaesthesia, ${ }^{2}$ Neurosurgery and ${ }^{3}$ Critical Care Medicine, Antwerp University Hospital, Belgium

Background and Goal of Study: The use of neuromuscular blocking agents (NMBAs), different reversal agents and their effect on respiratory outcome after surgery is still the subject of research and debate. Since the availability of sugammadex the use of a deep neuromuscular block (NMB), i.e. post-tetanic count of 1-2 on acceleromyography, has regained popularity. As part of a larger trial, we wanted to assess whether a deep neuromuscular blockade reversed with sugammadex resulted in similar inspiratory muscle activity when emerging from anesthesia compared to spontaneous reversal of NMB after a single dose of NMBA.

Materials and Methods: We used a

transcutaneous electromyography (EMG) method to evaluate both diaphragm (EMGdi) and intercostal (EMGic) muscle activity in a prospective, double-blind randomized controlled trial in patients receiving propofol-remifentanil anaesthesia. EMG activity was measured after the train-of-four (TOF) returned to $>90 \%$, in the interval between the onset of spontaneous breathing and extubation of the trachea. EMG amplitude peaks on inspiration were used. Data were compared with the Mann-Whitney U statistical test. Fig 1 shows the study protocol.



Figure 1: Study protocol

Results and Discussion: 32 patients were analyzed. Median EMGdi and EMGic were similar for both groups $(P=0.83$ and $\mathrm{P}=0.06$ ): spontaneous recovery from single dose NMBA median EMGdi 4.0 $\mu \mathrm{V}$ (IQR 2.0 - 7.9) and EMGic $2.3 \mu \mathrm{V}(1.3$ 4.6); deepNMB EMGdi 4.4 $\mu \mathrm{V}$ (IQR 1.9 - 7.5) and EMGic 1.8 $\mu \mathrm{V}$ (1.1 - 3.7). (see Fig 2 and 3)The use of a deep NMB did not influence the action of inspiratory muscles when emerging from general anesthesia in this study.
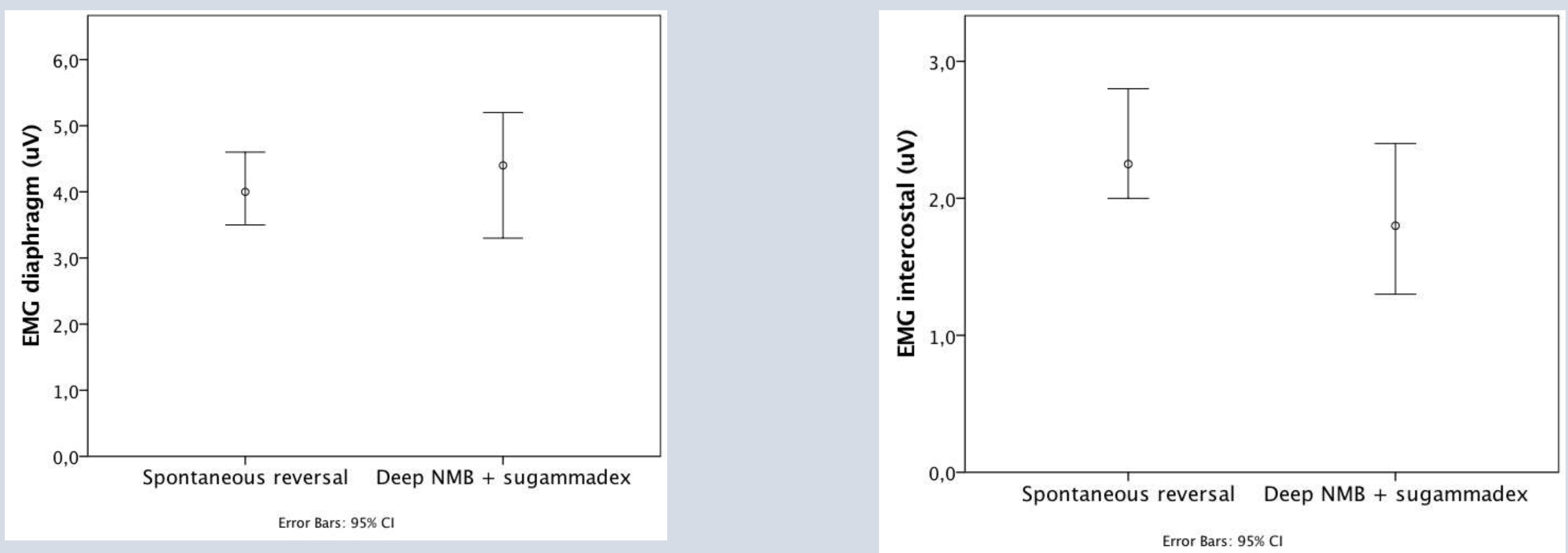

Figures 2 and 3: Diaphragm (Fig 2, left) and intercostal (Fig 3, right) EMG in spontanenous and sugammadex reversal groups

\section{Conclusion}

In this study, the use of a deep NMB reversed with sugammadex resulted in similar activity of inspiratory muscles compared to a single dose of NMBA and spontaneous recovery. This study suggests deep neuromuscular blockade and sugammadex reversal has no impact on postoperative respiratory safety. More studies are needed to evaluate the effect of sugammadex and neostigmine on respiratory muscle activity. 